\title{
El Instituto Torroja, edificio histórico: conservación y características de los materiales de sus fachadas
}

\author{
Torroja Institute, the historic building: conservation and \\ characteristics of the materials of its façades
}

M. P. LUXÁN, D. GASPAR, R. SOTOLONGO, M. T. SOLESIO y F. DORREGO Instituto de Ciencias de la Construcción Eduardo Torroja (CSIC)

Fecha de recepción: 23-IV-99

Fecha de aceptación: 19-V-99

ESPAÑA

\begin{abstract}
RESUMEN
El edificio del "Instituto de Ciencias de la Construcción", construido en 1951, constituye una de las obras singulares del ingeniero Eduardo Torroja. En el centenario de su nacimiento se ha elaborado este estudio, que se centra en los materiales de construcción de las fachadas del Instituto. De su análisis se desprende la importancia del diseño del proyecto original en la buena conservación de los paramentos exteriores.

La caracterización de los morteros de junta de las fábricas de ladrillo de los paramentos, mediante técnicas instrumentales (espectroscopia infrarroja, difracción de rayos $X$, microscopia electrónica de barrido con energías dispersivas de rayos $X$ ) revela que la calcita es el compuesto principal, con presencia de yeso -especialmente en zonas externas contaminadas- $y$ compuestos siliceos procedentes de la arena. Los resultados sobre las fachadas reflejan una buena compatibilidad entre los morteros de cal y los ladrillos.
\end{abstract}

\section{SUMMARY}

The "Instituto de Ciencias de la Construcción" building is one of the most singular engineering design done by Eduardo Torroja; it was started in 1951. This study focussed on the Institute façade-building materials has been carried out in the $100^{\text {th }}$ anniversary of his birth. The analysis has revealed that the design of the original project has been of a great importance for the good conservation of the external walls.

The pointing mortar characterization of its brick walls by instrumental techniques (infrared spectroscopy, X-ray diffraction, scanning electron microscopy with EDS) reveals that calcite is its main component, with gypsum presence in polluted external zones and silica compounds from the sand. The obtained results indicate a good compatibility between mortars and façade bricks.

\section{PLANTEAMIENTO}

Una de las obras singulares de Eduardo Torroja es el edificio que alberga el centro de investigación sobre la construcción y sus materiales y que hoy lleva su nombre, nombre con el que es también conocido en el ámbito internacional.

Con motivo del centenario de su nacimiento se ha querido reflejar la actualidad y permanencia de esta construcción, por su carácter histórico y símbolo de una época, desde el punto de vista de los materiales empleados en la ejecución de las fachadas. La construcción de este edificio fue profusamente detallada en el documento "Costillares es así", donde se recopilan los trabajos 
previamente publicados (1) (2) (3) sobre los aspectos constructivos y arquitectónicos del proyecto original y de las fases de la obra; sobre los materiales empleados se recoge su origen y tipología, mas no se incluyen datos sobre las propiedades de los morteros de junta y los ladrillos de las fachadas.

En este estudio, tras una breve descripción del edificio en sus inicios y de su reglamentación otrora elaborada, se analizan las características y prestaciones de los materiales de construcción de los paramentos de las fábricas de ladrillo y el estado de conservación de las fachadas en la actualidad.

\section{EL PROYECTO ORIGINAL DEL EDIFICIO}

Las obras del edificio que alberga el Instituto se comenzaron en 1951 tras 17 años de andadura de la primigenia Institución.
El proyecto fue dirigido por Eduardo Torroja, quien eligió para su emplazamiento un solar de $5 \mathrm{Ha}$ en un pequeño cerro situado en la zona Norte (finca El Bosque, en Chamartín de la Rosa) de la ciudad de Madrid (1).

El conjunto contemplaba un edificio central con cuatro cuerpos, una planta piloto donde quedaba instalado un horno para la fabricación de clínker y naves para ensayos (Fig. 1). Al ser concebido desde un principio como sede de un centro de investigación, el edificio no sólo albergaba despachos, biblioteca, sala de conferencias, capilla, sino también laboratorios para investigación química, física y el análisis de las propiedades mecánicas y el comportamiento de materiales y estructuras.

Visibles externamente, formaban parte también del proyecto original: el depósito elevado de agua, alojado en una torre revestida de material pétreo (Fig. 2), en el ala sur del edificio central; el comedor, en forma de rotonda, con cerramiento acristalado de apertura móvil orientada hacia

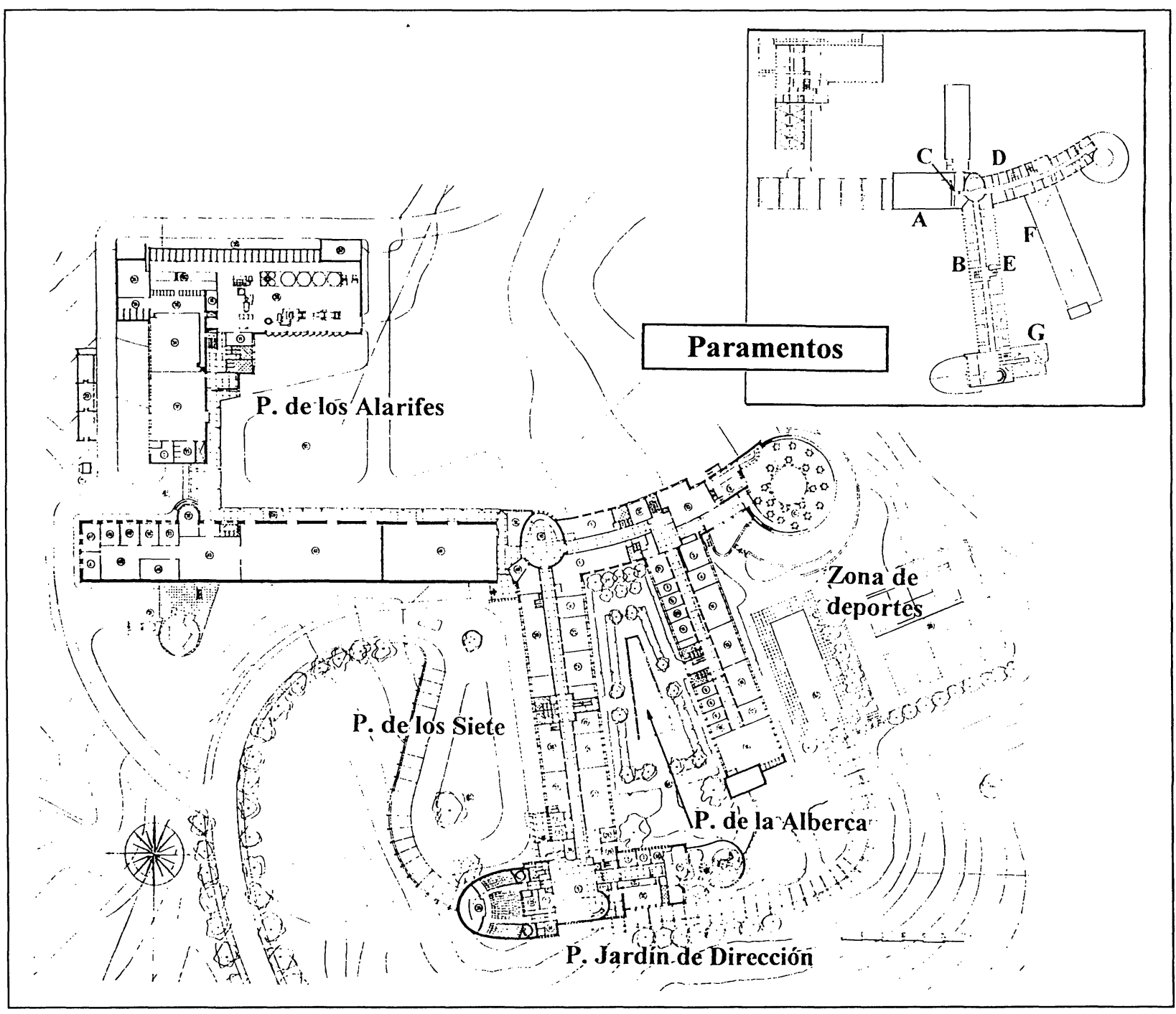

Figura 1.- Plano del Instituto Torroja (Echegaray, 1953). Plano actual (Minguez, 1999), con las zonas de análisis y toma de muestras. 


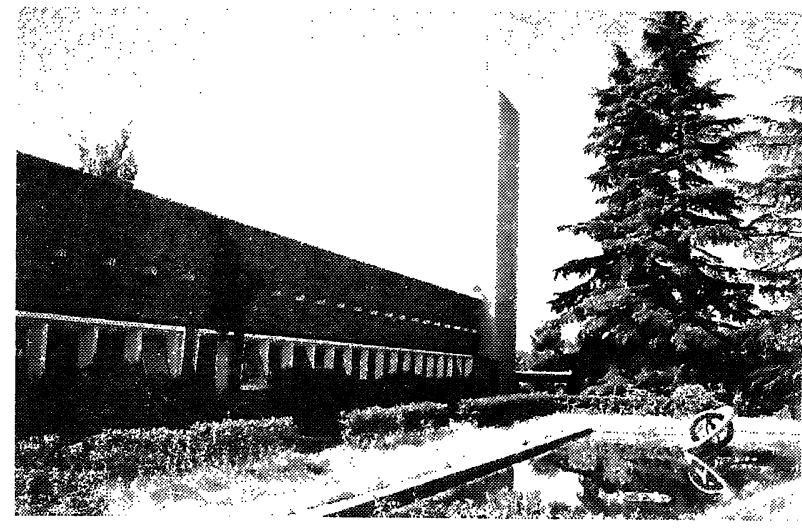

Figura 2.- Estanque de enfriamiento del agua de los grupos electrógenos. Nenúfares y una pieza escultórica conmemorariva de CEMCO contribuyen a la estética ambiental. Torre y fachada $\mathrm{F}$.

hacia el jardín; y el dodecaedro, diseñado originalmente como depósito del carbón para la calefacción, con una capacidad de $250 \mathrm{t}$, que, con los años, se ha convertido en señal de identidad del propio Instituto.

Las edificaciones están rodeadas de jardines, con espacios deportivos (tenis y piscina), lo que confieren unas características de habitabilidad singulares para un centro de investigación de este tipo. Por su peculiar diseño quedan delimitadas una serie de zonas o patios (P.) principales (Fig. 1) que reciben nombres concretos: el P. de los Siete, a la entrada con los aparcamientos; el P. de la Alberca es un tranquilo jardín de planta trapezoidal, con un estanque donde proliferan los nenúfares, resulta, así, un pequeño remanso en pleno centro del Instituto (Fig. 2); el P. de Alarifes, de acceso a los lugares de trabajo y con posibilidad de carga y descarga (Fig. 3); el Patio - Jardín de Dirección, con especies arbóreas importadas.

La construcción ha permanecido en su estructura original, sin modificaciones fundamentales en su exterior. Los cambios sufridos en el transcurso de los años no alteran, básicamente, el conjunto antaño edificado, sino que más bien incorporan espacios construidos a los ya existentes. Éste es el caso de las ampliaciones de la Planta de elaboración de Arenas, que, desde la época de E. Torroja, posee el Instituto de forma pionera y exclusiva para el control de la calidad de los cementos; actualmente prepara Arena Normalizada (Marca AENOR) para llevar a cabo la Certificación de los Cementos. Más tarde, se construyó otra nave de ensayos y, en los últimos años, también se han diseñado modernas instalaciones para salas de reuniones y aulas de formación, donde se siguen celebrando los Cursos de Estudios Mayores de la Construcción (CEMCO), que fueron instituidos en su origen por Eduardo Torroja, destinados a la cooperación con países de América del Sur y Central. Son obras dignas de señalar la construcción de la cubierta laminar exenta perfilada, a semejanza de las estructuras diseñadas por Torroja, situada

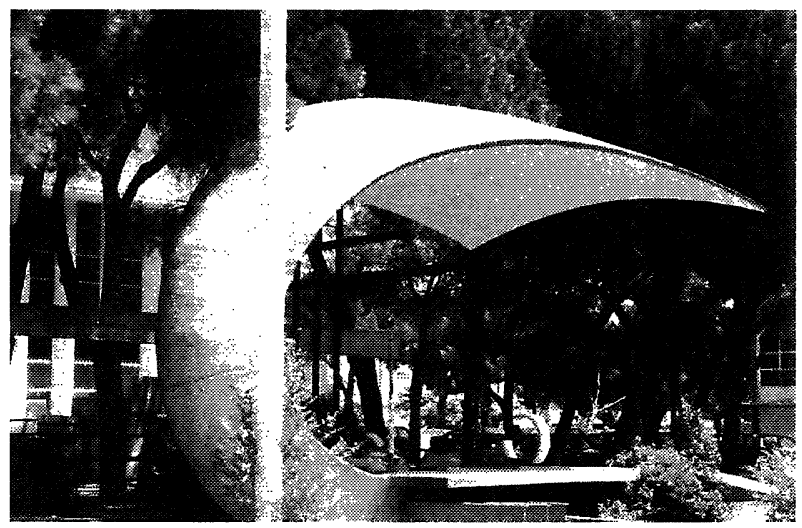

Figura 3.- Patio de los Alarifes.

en el Patio de Alarifes (Fig. 3) y también las piezas escultóricas incorporadas en los espacios abiertos (Fig. 2), que simbolizan el paso de los alumnos del CEMCO por el Instituto a lo largo de los XIV Cursos impartidos.

Las fachadas del Instituto mantienen su fisonomía y diseño original. El concepto y planteamiento en la selección de los materiales para la construcción de las fachadas ha contribuido a su buena conservación a lo largo del tiempo.

Ladrillo, piedra y morteros, han sido los materiales básicos empleados en la ejecución de las fachadas. Todos los paramentos, excepto la Sala de Conferencias y la nave de Talleres, son de ladrillo cerámico cara vista, con juntas horizontales y verticales rehundidas (Fig. 4).

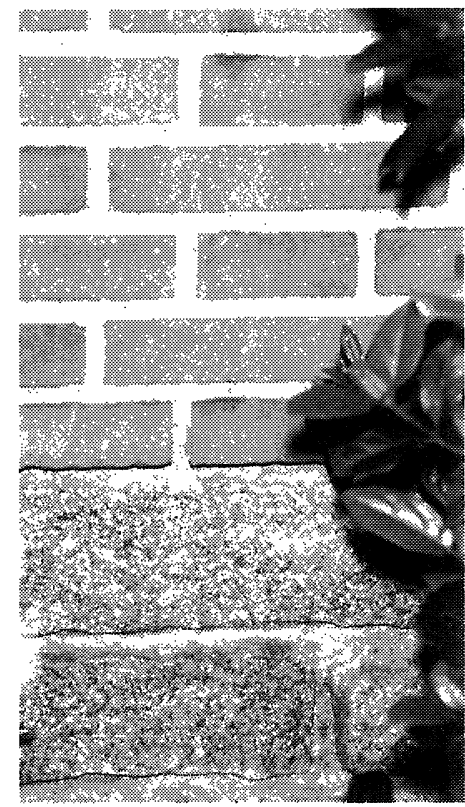

Figura 4.- Paramento de fachada (zona A). Detalle de la fábrica de ladrillos. 
En las fachadas se emplearon también sistemas constructivos propios, diseñando piezas prefabricadas para los marcos de las ventanas y las gárgolas a base de hormigón armado con cemento blanco (2).

El basamento de todas las fachadas lo constituye un zócalo de mampostería de granito; su variable dimensión en altura, en conexión con las variaciones de cotas de nivel del terreno, proporciona movilidad visual a las superficies.

Las reparaciones a lo largo de los años han tenido como objeto solventar los problemas de filtraciones del agua de lluvia y el atasco de los desagües debido a la acumulación de hojas de pino y suciedades. Entre las modificaciones realizadas al edificio se incluyen: la sustitución de las ventanas en las fachadas y de la cubierta en el comedor, la construcción de la entrada a la autopista M-30 en zonas ajardinadas y la instalación de gases para los equipos de caracterización de materiales. En este último caso, la necesidad de proceder en cumplimiento de las normativas de seguridad, ha motivado la incorporación en el exterior del edificio de pequeñas casetas para alojar las bombonas, adosadas a las fachadas en los lugares anexos a los laboratorios correspondientes (Fig. 2).

\section{LA REGLAMENTACIÓN SOBRE LOS MATERIALES EN LA ÉPOCA DE CONSTRUCCIÓN DEL EDIFICIO TORROJA}

En la época de la construcción del Instituto el interés por la calidad de los materiales iba en aumento y se habían ya desarrollado numerosos estudios que, en España, se plasmaron principalmente en pliegos y recomendaciones.

En el año 1951, fecha de la edificación, existían los siguientes documentos (4):

- El "Pliego General de Condiciones para la Recepción de los Aglomerantes Hidráulicos en las Obras de Carácter Oficial" (R.O. de 25 de febrero de 1930).

- El Pliego General de Condiciones Técnicas para la Edificación" de 1948, que recoge aspectos sobre las CALES (definiciones; clasificación; propiedades)y sobre los YESOS (definición; tipos, clasificación; empleo y características).

- La Norma NELC 3.03a "Análisis químico del yeso y productos afines", publicada en 1950 por el Laboratorio Central de Ensayo de Materiales de Construcción, que está basada en la normativa ASTM.

Se constata así la existencia de documentos de referencia para verificar la calidad de los materiales de construcción (cementos, cales y yesos) durante la primera mitad del siglo XX. Es, desde estos momentos, -y especialmente en los últimos años con las directrices europeas- cuando la normativa española (normas UNE) y europea se han desarrollado con mayor amplitud.

\section{LAS FACHADAS Y SUS MATERIALES A FINALES DEL SIGLO XX}

Las fachadas del edificio Torroja poseen, en líneas generales, un buen estado de conservación, presentando daños puntuales, debidos, principalmente, a efectos ocasionados por la lluvia, con las correspondientes escorrentías y deslavado superficial de los morteros de las juntas en las fábricas de ladrillo (Fig. 5).

La buena conservación de las fachadas se debe, en gran parte, al diseño del proyecto original y, en concreto, a la construcción de un zócalo de granito perimetral sobre el que luego se asienta la fábrica, impidiendo su degradación por efecto de la humedad ascendente procedente del suelo lo cual constituye uno de los principales problemas en la conservación de edificios (5). También es debida a la calidad de los materiales seleccionados y a la ejecución de la obra.

Se observan diferencias en la conservación de los materiales localizados en zonas umbrías, con mayor retención de humedad y donde el crecimiento biológico ha tenido lugar (musgos,...), respecto a aquéllos que se encuentran en lugares preferentemente soleados.

Atendiendo a cada uno de las tipologías de materiales existentes, se pueden efectuar las siguientes observaciones:

\section{Ladrillos}

La calidad del ladrillo de fachada es superior a la del ladrillo empleado en interiores (5), con una temperatura de cocción mayor en su fabricación y, por ello, con mejor comportamiento con relación a la humedad y al movimiento de agua en el paramento. Sus dimensiones son similares en todas las fachadas (zonas A, B, C, E, F, ... de la figura 1), alcanzando los valores de $(25 \times 12 \times 5) \mathrm{cm}$.

No se observa deslaminación, ni pérdidas superficiales de los ladrillos en las zonas donde se han producido las escorrentías(Fig. 5). Su estado de conservación es bueno. Hay algunos lugares puntuales donde se han producido humedades desde el interior del edificio, como es el caso de la entrada a la conserjería en el soportal de acceso, y se observan daños en los ladrillos por contaminación interna de sales, que han producido pérdidas de cohesión en la masa de los ladrillos. 


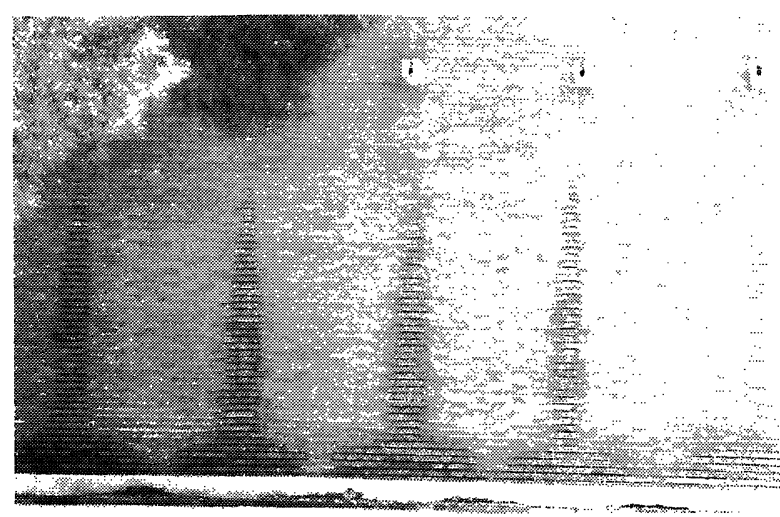

Figura 5.- Gárgolas con canalón en el Patio de los Alarifes. Escorrentías y salpiqueo en el alero de la cubierta del corredor.

\section{Materiales pétreos}

El antiguo depósito de agua, recubierto de placas de piedra de Novelda, ha sido toscamente reparado. El deterioro mayor en este material, que se refleja en deslaminación y pérdida de la superficie abujardada, se debe al agua de lluvia y se manifiesta, principalmente, en la zona de entrada al Patio de la Alberca, bajo el pórtico. La piedra de Colmenar, que se encuentra en las puntas de diamante, ofrece mejor conservación que la piedra de Novelda.

El zócalo de granito ofrece, en general, un buen estado de conservación. Su tamaño es irregular, aunque se mantiene la dimensión de las hiladas, y la talla de cantería le confiere un aspecto rústico.

\section{Morteros}

Los morteros que se encuentran en la fábrica de ladrillos de las fachadas ofrecen un aspecto homogéneo. Sin embargo, cabe señalar dos tipos de ejecución de la fábrica que marcan localizaciones concretas del edificio. En ambas se mantiene un mismo tamaño del tendel, con un valor aproximado en torno a $1,5 \mathrm{~cm}$, pero se diferencian en que el espesor de las llagas es, en unos casos similar al tendel, con valores de $1,5 \mathrm{~cm}$ (zonas A, B, C, E, F, G: Fig. 1), mientras que en el Patio de Alarifes y en las edificaciones colindantes la colocación de los ladrillos se hizo a hueso.

Los morteros de junta de las fachadas antiguas no presentan deterioro, salvo si se encuentran en aquellas zonas donde ha habido problemas de humedad o están en el camino de evacuación de las aguas de escorrentías.

En las partes que se localizan por debajo de las gárgolas el daño en los morteros se desarrolla en forma triangular en la superficie de la fábrica de ladrillo, según los lugares por los que discurre el agua de lluvia (Fig. 5). El daño sufrido ha ocasionado la pérdida de la capa superficial del mortero en el acabado de las juntas, dejando con ello al descubierto el mortero de la junta de la fábrica de ladrillo. Al proseguir el fenómeno, se ha producido un deslavado del mortero, con pérdida de su masa aglomerante y, más tarde, con la pérdida, también, de la totalidad del mortero, a una profundidad de unos $2 \mathrm{~cm}$.

En el soportal de entrada a la conserjería, con la aparición de humedades desde el interior, los morteros muestran arenización y pérdida de cohesión, al igual que los ladrillos dañados en dichos lugares.

En las zonas umbrías y con mayor retención de humedades los morteros experimentan crecimiento biológico.

\section{CARACTERIZACIÓNDE LOSMATERIALES EXISTENTES DE LAS FACHADAS}

La caracterización de materiales se ha efectuado mediante métodos no destructivos, aplicados directamente en la fachada y además se han extraído muestras para evaluar las características de los morteros y de los ladrillos. Este estudio se ha efectuado en cada una de las fachadas antiguas del edificio (Fig. 1).

Las propiedades mecánicas de los materiales de las fábricas (ladrillo y mortero) se han evaluado in situ por medio del martillo Schmidt, realizando el correspondiente calibrado previo en el laboratorio para cada una de las tipologías de materiales a caracterizar. Los resultados se han obtenido como media de más de 100 medidas en cada una de las zonas de estudio establecidas para las fachadas del edificio. Los valores medios obtenidos se encuentran en la Tabla I y se expresan como equivalencia a la resistencia a compresión.

Los ladrillos presentan una gran similitud en los resultados obtenidos (Tabla I) y la mayor diferencia se obtuvo al realizar medidas esclerométricas comparativas entre ladrillos húmedos (35,2 $\mathrm{MPa})$ y ladrillos secos (38,0 MPa) (Fig. 6).

Las propiedades mecánicas de los morteros de junta de la fábrica de ladrillo se recogen igualmente en la Tabla I. La resistencia de los morteros es baja (valor medio: 2,2 $\mathrm{MPa}$ ) como corresponde a su composición de morteros de cal; hay mayor dispersión en los datos obtenidos que en el caso de los ladrillos, debido, no sólo a estas mezclas preparadas, sino también a que la medida se efectúa para valores muy bajos y el equipo está cercano a sus límites de detección.

Las muestras de mortero se han extraído de las juntas de las fábricas de ladrillo, teniendo en cuenta las distintas 
Valores medios equivalentes a la resistencia a compresión de los ladrillos de la fábrica y morteros de junta de las fachadas del edificio Torroja en las diferentes zonas de estudio

\begin{tabular}{|c|c|c|}
\hline \multirow{2}{*}{$\begin{array}{l}\text { PARAMENTOS } \\
\quad \text { (Fig. 1) }\end{array}$} & \multicolumn{2}{|c|}{$\begin{array}{l}\text { VALOR MEDIO EQUIVALENTE A LA } \\
\text { RESISTENCIA A COMPRESIÓN (MPa) }\end{array}$} \\
\hline & LADRILLOS & MORTEROS \\
\hline Zona A (bajo soportal) & 38,1 & 2,1 \\
\hline Zona A (intemperie) & 38,7 & 1,8 \\
\hline Zona B (bajo soportal) & 39,0 & 1,1 \\
\hline Zona B (intemperie) & 39,4 & 2,6 \\
\hline Zona $\mathrm{C}$ (interior) & 38,6 & 2,4 \\
\hline Zona D (intemperie) & 40,1 & 2,7 \\
\hline Zona E (intemperie) & 38,5 & 1,9 \\
\hline Zona F (intemperie) & 38,0 & 2,6 \\
\hline Zona G (intemperie) & 38,4 & 2,3 \\
\hline MEDIA DE LOS VALORES MEDIOS & 38,7 & 2,2 \\
\hline
\end{tabular}

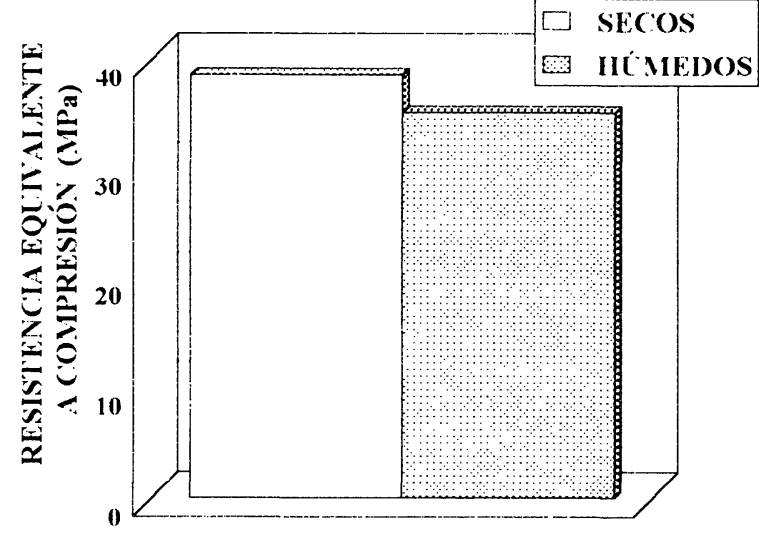

Figura 6.- Resistencia a compresión equivalente de los ladrillos de fachada (zona F).

orientaciones y localizaciones en el Instituto (Fig. 1), así como las partes internas y externas de las juntas.

De los análisis se desprende que los morteros de las juntas fueron elaborados con una mezcla de cal y arena (Figs. 7, 8 y 9), en una proporción, en peso, en torno a 1:4 (valor medio). Este valor se encuentra en el rango de dosificaciones obtenido en otros estudios sobre morteros de junta de edificios históricos, que proporcionaban valores entre $1: 2$ y $1: 5,(6)$.

Los morteros estudiados tienen una composición similar, las variaciones que se perciben parecen haberse originado durante la preparación de la mezcla en obra, debido a incorporaciones ligeramente variables de arena $y$, por tanto, partículas finas contenidas. En la composición mineralógica de la fracción fina del mortero se identifica, mediante espectroscopía de absorción infrarroja (Fig. 7) y difracción de rayos X (Fig. 8), el carbonato cálcico (C), como componente mayoritario, yeso $(G)$ y diferentes proporciones de cuarzo y silicatos ( $\mathrm{Si}$ ) (feldespatos, caolinita,...). La microestructura del mortero se ha estudiado mediante microscopía electrónica de barrido, observando las placas de calcita y corroborando la composición previamente obtenida al aplicar las energías dispersivas de rayos $\mathrm{X}$ (Fig. 9).

La tonalidad de los morteros es blanca y, en todos los casos, se detectan cantidades de yeso en bajo porcentaje (Figs. 7, 8 y 9), tanto en el interior de la junta cómo en la capa exterior. Cabe señalar como en la zona A de la fachada, situada en la puerta de entrada del Instituto con acceso de vehículos, el exterior de la junta muestra mayores niveles de yeso (Fig. 7, 1MORT), lo que indicaría la sulfatación del mortero de cal por efecto de la contaminación externa. La composición de la capa superficial de la junta (zona A) se ha comparado con las muestras del mismo paramento en lugares del soportal (Fig. 7, 3 MORT - zona A), más resguardados del ambiente exterior, e incluso en el interior del edificio, donde aún se ha mantenido la decoración con ladrillo visto (Fig. 7, 9 MORT - zona C), verificando el mismo hecho, ya que su contenido en yeso en estas localizaciones era menor.

Los áridos de tipo silíceo empleados en los morteros de junta son típicos en los morteros de la época en la provincia de Madrid. Sus curvas granulométricas son similares en los morteros extraídos en las diferentes 


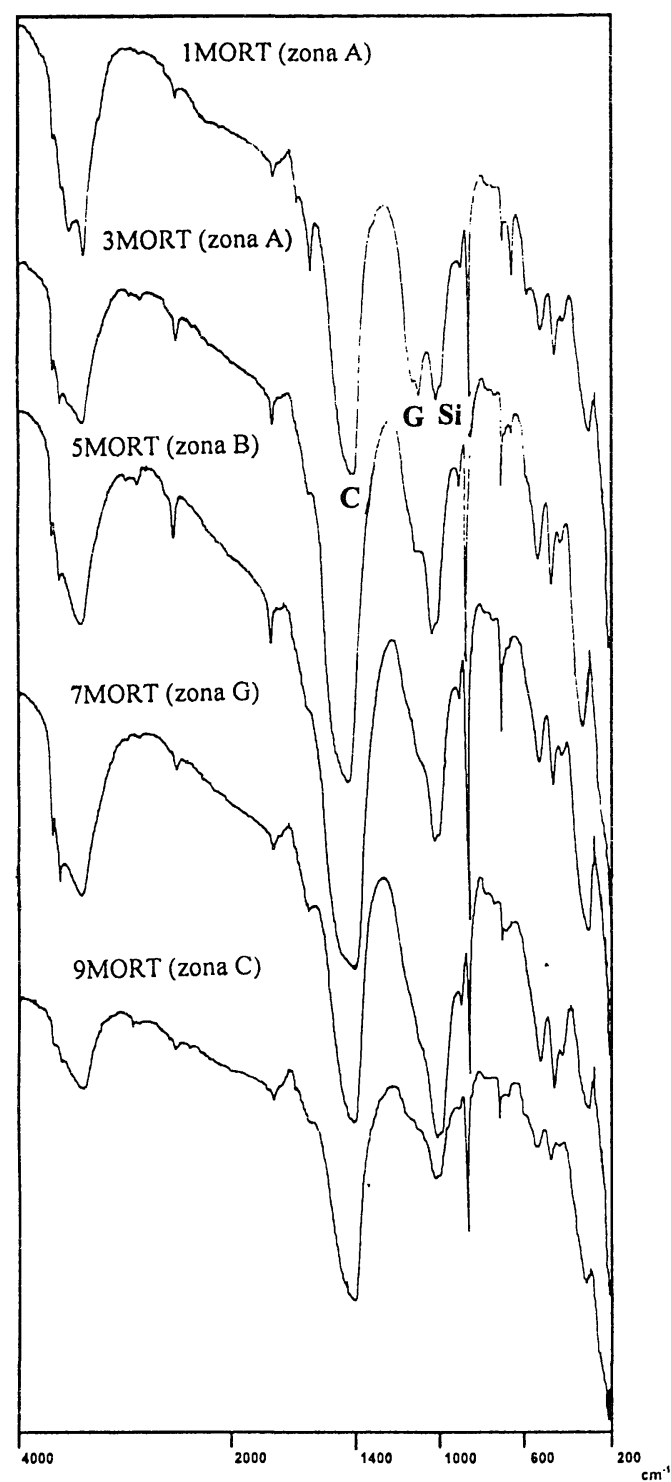

Figura 7.- Caracterización de los morteros de junta mediante espectroscopía infrarroja.

localizaciones (Fig. 10), con valores máximos inferiores a $3 \mathrm{~mm}$ y tamaños más frecuentes entre 0,16 y $0,5 \mathrm{~mm}$.

En el exterior de las juntas los morteros que rematan la fábrica presentan una fina capa con árido fino y en una proporción (1:1), más baja que el mortero del interior.

Finalmente, se constata la compatibilidad de los materiales empleados en las fábricas de ladrillo sin deterioro en los ladrillos a causa de los morteros (ausencia de cristalizaciones internas por efecto de movilidad salina, rotura de los ladrilllos por diferencias en las resistencias mecánicas y excesiva dureza de los morteros,...) y viceversa; actualmente se presenta una buena adherencia entre los ladrillos y el mortero de cal de las juntas (Fig. 4) y no se observan daños (fisuras de retracción,...) en la zona de adherencia para las muestras

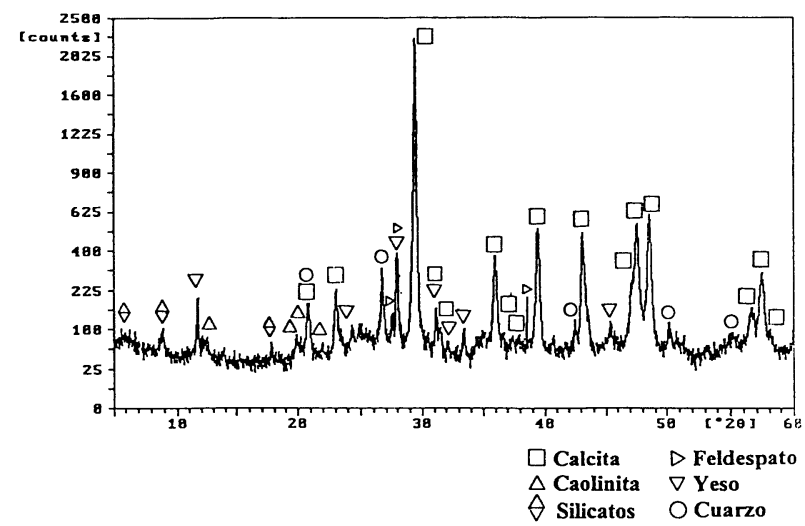

Figura 8.- Composición mineralógica de los finos del mortero de junta (difracción de rayos X de la muestra 3 MORT-zona A).

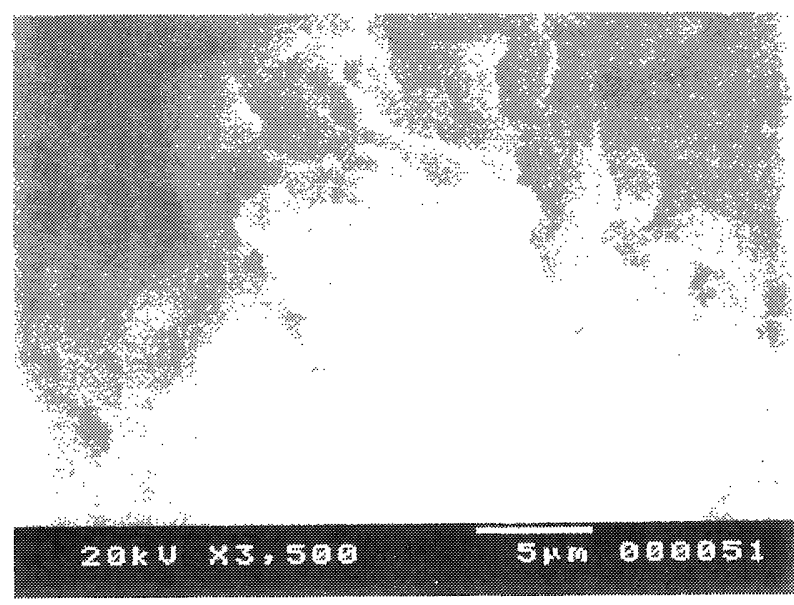

Figura 9.- Microestructura del mortero de junta (micrografia de la muestra 2 MORT-zona A, mediante microscopia electrónica de barrido).

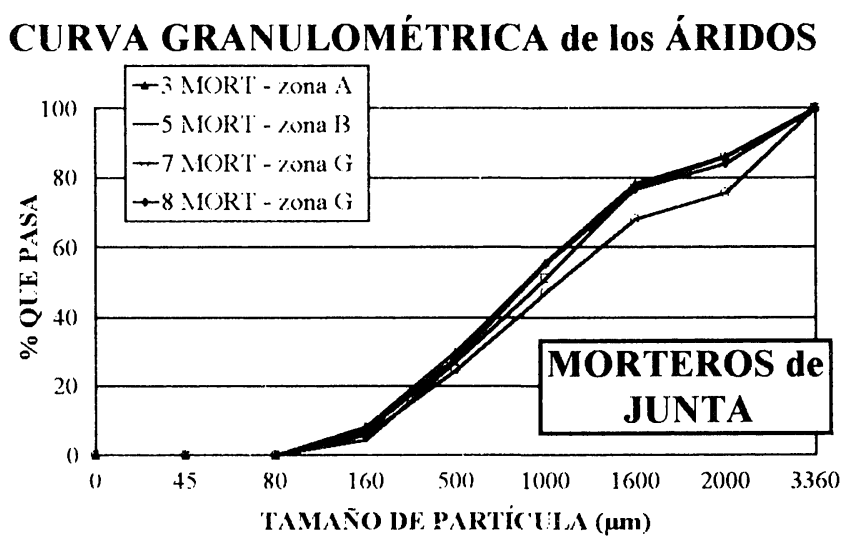

Figura 10.- Curvas granulométricas de los áridos de los morteros de junta de los paramentos exteriores del edificio Torroja. 
estudiadas, lo que también es indicativo de una puesta en obra de los ladrillos correcta (humectación previa a su colocación,...).

\section{AGRADECIMIENTO}

Los autores agradecen la financiación de los estudios sobre las características de los morteros de junta en las fábricas de ladrillo, que se encuadran en el Proyecto Europeo CE-ENV4-CT98-0706 "Maintenance of pointing in historic buildings: Decay and replacement".

\section{BIBLIOGRAFÍA}

(1) NADAL, J.: "El Instituto Técnico de la Construcción y del Cemento", Informes de la Construcción, n 55, 10 pp. (1953) en "Costillares es así"
(2) ECHEGARAY, G., BARBERO, M.: "Composición Arquitectónica", Informes de la Construcción, n56,20 pp. (1953) en "Costillares es asi"

(3) ARREDONDO, F.: "Organización de Obra", Informes de la Construcción, n59, 10pp. (1954)en “Costillares es así".

(4) GASPAR, D. y col.: "Los materiales en la época de construcción del edificio Torroja a través de su reglamentación", Materiales de Construcción (en prensa)

(5) CSTC.: "Lehumidité dans les constructions. Particularites de l'humidité ascencionnelle”, NIT n² 210,55 pp. (1998)

(6) BARONIO, G., BINDA, L.: "Experimental approach to a procedure for the investigation of historic mortars", Proc. $9^{\text {th }}$ Int. Brick/Block Masonry Conf., Berlin, 1397-1405. (1991)

\begin{tabular}{|c|c|c|}
\hline 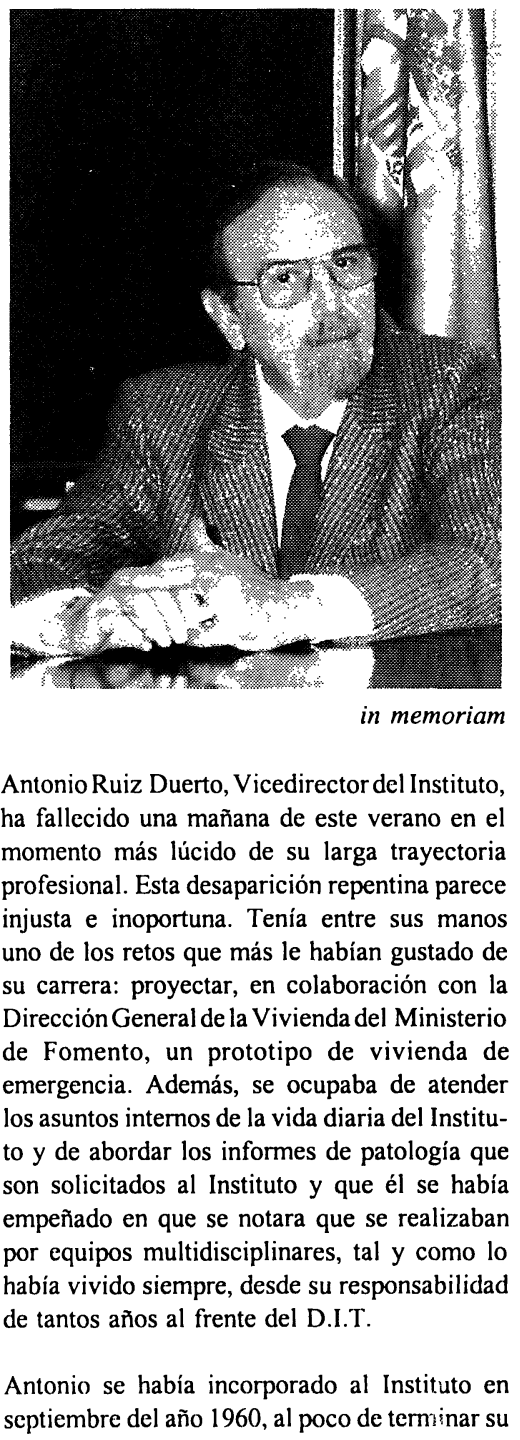 & 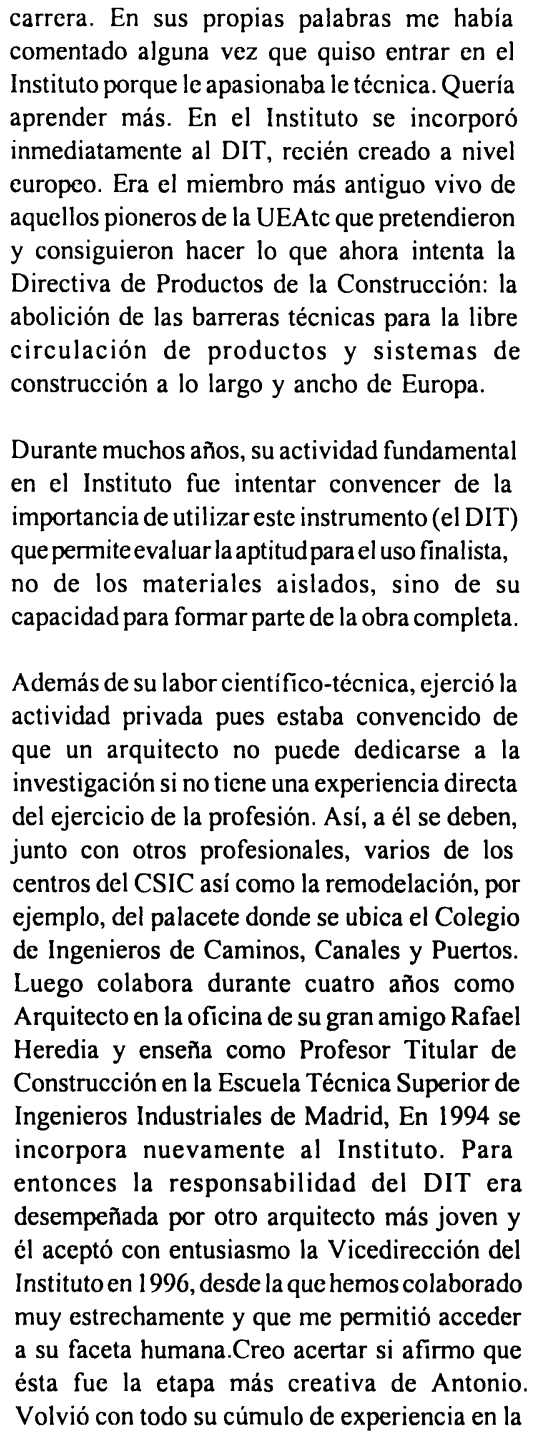 & 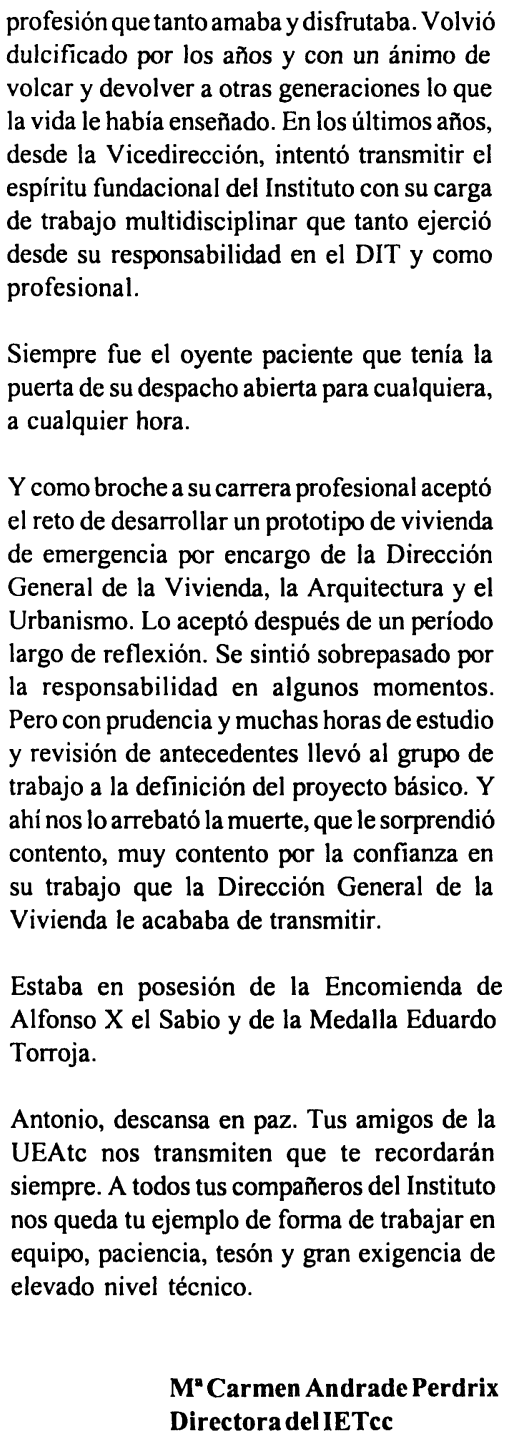 \\
\hline
\end{tabular}

